\title{
Cross-link debonding in actin networks - influence on mechanical properties
}

\section{Björn Fallqvist, Artem Kulachenko and Martin Kroon*}

Department of Solid Mechanics, Royal Institute of Technology,

Teknikringen 8, 10044 Stockholm, Sweden

Email: bfa@kth.se

Email: artem@kth.se

Email: martinkr@kth.se

*Corresponding author

\begin{abstract}
The actin cytoskeleton is essential for the continued function and survival of the cell. A peculiar mechanical characteristic of actin networks is their remodelling ability, providing them with a time-dependent response to mechanical forces. In cross-linked actin networks, this behaviour is typically tuned by the binding affinity of the cross-link. We propose that the debonding of a cross-link between filaments can be modelled using a stochastic approach, in which the activation energy for a bond is modified by a term to account for mechanical strain energy. By use of a finite element model, we perform numerical analyses in which we first compare the model behaviour to experimental results. The computed and experimental results are in good agreement for short time scales, but over longer time scales the stress is overestimated. However, it does provide a possible explanation for experimentally observed strain-rate dependence as well as strain-softening at longer time scales.
\end{abstract}

Keywords: actin; cross-link; filament; network; debonding; stress; strain-rate; stochastic.

Reference to this paper should be made as follows: Fallqvist, B., Kulachenko, A. and Kroon, M. (2015) 'Cross-link debonding in actin networks - influence on mechanical properties', Int. J. Experimental and Computational Biomechanics, Vol. 3, No. 1, pp.16-26.

Biographical notes: Björn Fallqvist received his Master's degree at Royal Institute of Technology, studying Mechanical Engineering. In 2011, as a PhD student he started research of cellular mechanics with an emphasis on the cytoskeleton. Currently, he is investigating the possibility to use constitutive models developed in a continuum framework to model the elastic and viscoelastic characteristics of the cytoplasm.

Artem Kulachenko is an Associated Professor at the Department of Solid Mechanics at the Royal Institute of Technology (KTH). He received his PhD in 2006 for the thesis on mechanics of paper webs written at the Mid Sweden University. He worked for three years as a Senior Researcher at KCL and later at the Technical Research Centre of Finland (VTT) working primarily with mechanics of paper products. His research interests include micromechanics of fibre-based materials, dynamics of axially moving materials and vehicle dynamics. 
Martin Kroon is an Associated Professor at the Department of Solid Mechanics at the Royal Institute of Technology $(\mathrm{KTH})$ in Stockholm. He received his $\mathrm{PhD}$ in 2003 at the Department of Solid Mechanics at KTH. His research interests include biomechanics of biological soft tissues, constitutive modelling of metals and polymers, and fracture mechanics.

\section{Introduction}

In the context of a mechanical approach to cell biology, there is a close relationship between cellular function and mechanical properties. Cells in a diseased state can exhibit a significantly altered mechanical response to external forces. This allows them to behave abnormally, promoting progression of the pathology. For example, in the case of cancer cells, reduced cell stiffness and adhesion strength (Rönnlund et al., 2013) promotes metastasis.

The most important part of the intracellular structure that allows the cell to respond to mechanical signals is the interconnected network of polymerised protein filaments called the cytoskeleton. This network determines the mechanical properties of the cell, functioning as both a passive mechanical structure and an entity that actively partakes in biological processes such as cell migration.

There are three major types of protein filaments in the cytoskeleton - actin filaments, intermediate filaments and microtubules. Each of these filaments is associated with a specific type of protein that may be added to or removed from a filament. This allows for remodelling of the cytoskeleton in response to signals such as external mechanical forces.

Actin filaments (F-actin) are formed from polymerisation of globular actin (G-actin). These filaments are crucial for a wide range of cellular functions and structural properties - for example, bundles of actin (filopodia) act to push the lamellipodium forward during cell migration while actin filaments bundled into stress fibres anchor the cell to the extra cellular matrix (ECM).

Actin binding proteins (ABPs) are cross-linking proteins, which regulate the architecture of the filamentous actin network. For example, $\alpha$-actinin favours the formation of bundles of filaments while the hinge-like structure of filamin promotes the formation of three-dimensional cross-linked networks, thought to provide much of the cytoplasmic stiffness. This network has the characteristics of a viscoelastic solid, in which the binding affinity of the ABP influences the macroscopic mechanical properties (Wachsstock et al., 1993, 1994). To predict the mechanical response of these actin networks a homogenised continuum-based approach, in which the network is assumed to behave as a continuous solid, can be utilised. We have previously proposed such a phenomenological chemo-mechanical constitutive model, in which the strain energy function of the material is assumed to be proportional to the concentration of cross-linked filaments (Fallqvist and Kroon, 2012).

Another option is to utilise tools such as finite elements to create a discrete numerical model. The influence of network geometrical and material parameters on the mechanical response and deformation field can then be more directly assessed. This approach was taken in previous work (Fallqvist et al., 2014; Onck et al., 2005; Huisman et al., 2007), demonstrating the influence of network parameters such as filament density on the mechanical response. Kim et al. (2011) performed Brownian dynamics simulations of 
actin networks, finding a distinct strain-rate dependence in which unbinding and unfolding events of cross-linking proteins was dominating the response at low and high shear rates, respectively. Abhilash et al. $(2012,2014)$ performed numerical finite element simulations of discrete networks with stochastic unbinding of cross-links.

In this paper, we utilise a somewhat similar method approach to model cross-link unbinding, but differ in that we do not use the well-known Bell's (1978) equation in terms of crosslink force and interaction length to modify the dissociation rate. Rather, we use Arrhenius' law to define the debonding kinetics in terms of an activation energy per bound cross-link, as this results in a more general approach - the strain energy need not only include the tensile force.

Figure 1 Domain of two-dimensional network model

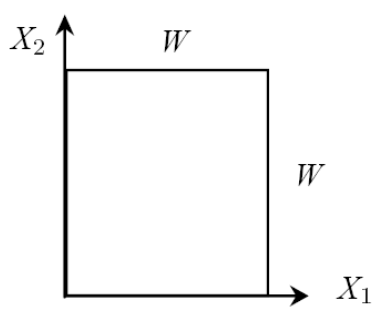

\section{Network model}

\subsection{Network model}

For details on this section, see work performed previously (Fallqvist et al., 2014). The software ANSYS was used for generating the geometrical network model, and the numerical computations were performed using a custom code integrated into ANSYS with user programmable features. A quasi-static implicit time integration solver was used.

We define a network region representative of a larger periodic structure in the form of a square network cell with length $W$, see Figure 1 . To generate the filaments constituting the network, we compute the network mass from an assumed concentration of $24 \mu \mathrm{M}$, the monomer mass and an assumed network thickness (here set to $0.02 \mu \mathrm{m}$ ). With the mass per filament easily computed with a known monomer-to-filament ratio (Biron and Moses, 2004), filaments are seeded at random positions and orientations until the sum of filament masses is equal to the network mass. Periodicity of the network is accounted for by cutting filaments extending over the edges and repositioning the cut sections to achieve a periodic structure. Each filament is discretised into 50 linear elastic and isotropic Euler-Bernoullis beam elements.

To ensure that the cell size $W$ is adequately large as to not influence the computed results, we refer to previous work (Fallqvist et al., 2014), where we performed studies on the influence of cell size. In light of this, we let $W=10 \mathrm{~L}$. It is assumed that no thermal fluctuations influence the filament shape, and they are generated with a perturbed straightness to elude local buckling instabilities. Further, all free ends are removed to improve convergence and reduce computational effort. From typical properties of actin filaments found in the literature (Furuike et al., 2001; Kamm and Mofrad, 2006; Gardel 
and Nakamura, 2006; Gardel et al., 2004, 2008), we choose to use a diameter of $6 \mathrm{~nm}$ and Young's modulus of $1.8 \mathrm{GPa}$. The filament length of this baseline model - the reference case to compare influence of parameter variation with - is set to what is typically found experimentally in reconstituted actin networks (Kasza et al., 2010; Xu et al., 2000; Nunnally et al., 1980), $15 \mu \mathrm{m}$. Of course, not all filaments can be expected to have an equal length - an exponential distribution is most commonly found in reconstituted networks. However, this has been found previously to not affect the qualitative behaviour of the network response (Fallqvist et al., 2014).

The free ends of the beam elements are removed to reduce computational effort. An example of a generated network model can be seen in Figure 2.

Figure 2 Example of generated finite element model (see online version for colours)

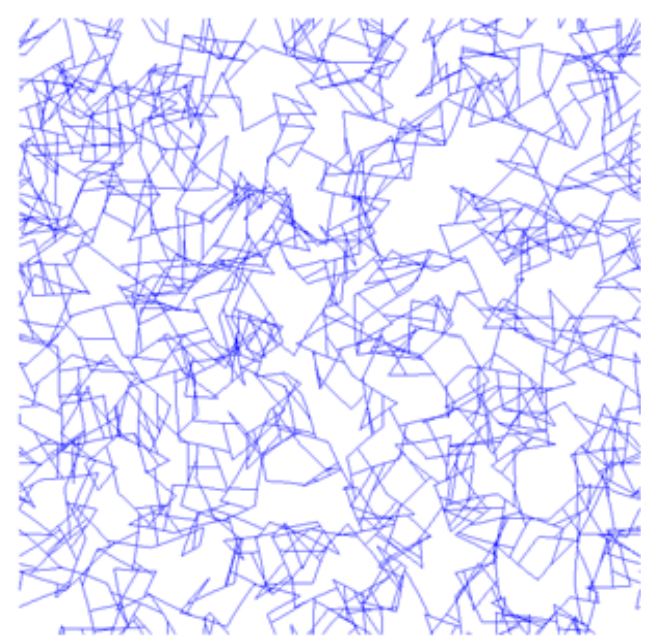

Note: The free dangling ends of the beams are removed to improve convergence.

\subsection{Cross-link model}

In actin networks, the rich variation of possible microstructures can be attributed to the multitude of available cross-linking proteins, as well as chemical factors such as the relative concentration of these proteins. We focus here on a network of cross-linked filaments without bundles, shown schematically in Figure 3. To model the influence of these cross-links and their properties on the network response, spring elements with zero initial length were created at intersection of filaments. The stiffness of these spring elements was defined as a hyperbolic function based on the deformed position of the nodes according to

$$
f_{b}(u)=C_{1} \cdot \sinh \left(C_{2} \cdot u\right)
$$

where $C_{1}$ and $C_{2}$ are parameters and $u$ is the relative displacement between the nodes. This relationship is chosen because it can replicate the mechanical response of the unfolding of cross-linking proteins reasonably well, while being simple to implement in the finite element code. For our baseline model we choose parameters to approximately 
reproduce experimental data of $\alpha$-actinin unfolding (Rief et al., 1999), resulting in $C_{1}=1.2 \cdot 10^{-5} \mu \mathrm{N}$ and $C_{2}=22 / \mu \mathrm{m}$.

Figure 3 Network of cross-linked filaments (see online version for colours)

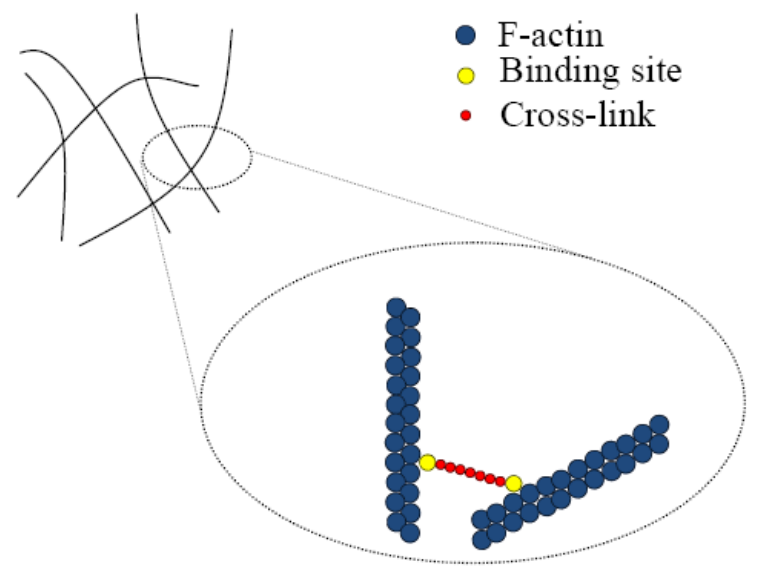

Figure 4 Bound state for cross-linked molecules

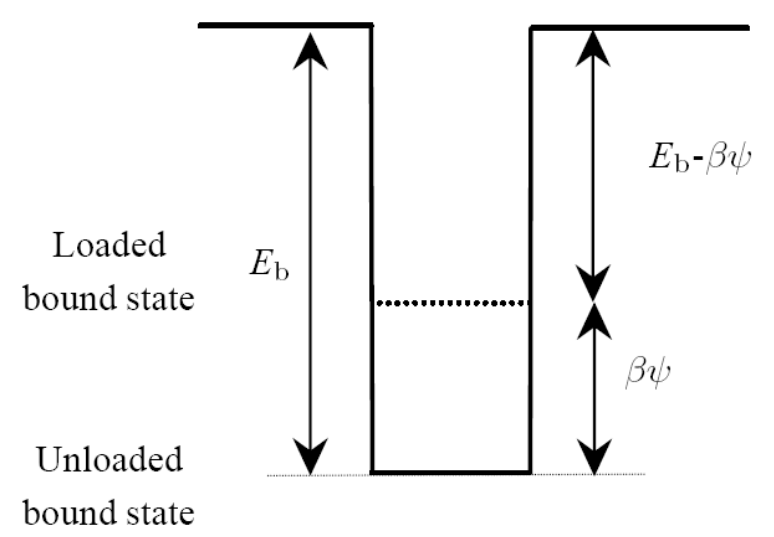

Note: To escape the potential well in the loaded state, the energy required to break the bond is $E_{b}-\beta \psi$.

The dynamic nature of cross-links can be traced back to its thermal exchange with the environment. A bound cross-link becomes trapped in an energy well of depth $E_{b}$ (defined as energy per $\alpha$-actinin bond), see Figure 4 . The stochastic nature of the bonds can be accounted for by considering the state of a bound cross-link in a probabilistic sense, according to

$$
p_{r}=A \cdot \exp \left(-\frac{E_{r}}{k_{B} T}\right),
$$


where $p_{r}$ is the probability of the cross-link to be found in a state with energy $E_{r}, k_{B}$ is Boltzmann's constant and $T$ the temperature. The factor $A$ is a pre-factor representing the number of collisions that might result in a reaction, which is here set to unity. This equation is similar to the well-known Bell's equation, except it is expressed in terms of activation energy, and not molecular force and interaction length.

Applying equation (2) to a bound cross-link according to Figure 4, the probability $\Delta p$ of a cross-link debonding during a short time interval will be

$$
\Delta p=\Delta t \cdot \exp \left(-\frac{\left(E_{b}-\beta \psi\right)}{k_{B} T}\right)
$$

where $\Delta t$ is the current time increment. The coefficient $\beta$ should be interpreted as a coefficient of free bonding energy, a fraction of the strain energy $\psi$ available to break bonds. A logic for implementing this in the finite element code is straightforward - for each created cross-link and in each time step, $\Delta p$ is compared to a random number $r$. If $\Delta p>r$ the element is deactivated for the subsequent steps, simulating a debonding event between filaments. The time increment $\Delta t$ was ensured to be small as to not influence the results, verified by performing identical computations of decreasing time step size. The bond energy $E_{b}$ is set to $2 k_{B} T$ (Biron and Moses, 2004). Rebinding of cross-links is not considered, and as such the model is an initial approach to studying the influence of cross-link debonding at an initial stage.

\subsection{Numerical implementation}

The indices on variables such as strains and stresses refer to the coordinate axes spanning the orthogonal frame in Figure 1.

For the nodes corresponding to $X_{1}=0$ and $X_{1}=W$, periodic boundary conditions were enforced - both translational and rotational degrees of freedom were coupled. For the nodes at $X_{2}=0$ and $X_{2}=W$, only the rotational degrees of freedom were coupled. The nodes at the top of the cell were given prescribed horizontal displacements when shearing the network with shear strain $\varepsilon_{12}$, and the vertical degrees of freedom were coupled.

To alleviate problems related to instabilities during debonding events, artificial stabilisation in the form of viscous forces were introduced. These were ensured to be small enough as to not affect the results.

The shear stress $\tau_{12}$ is computed as the sum of the extracted nodal reaction forces divided by the projected network area (in the thickness direction, assuming a network thickness $t$ ). For comparisons with experiments, we use our previously defined baseline model, i.e., filament length $L=15 \mu \mathrm{m}, \beta=1, W / L=10, E_{b}=2 k_{b} T, T=298 \mathrm{~K}$, $C_{1}=1.2 \cdot 10^{-5} \mu \mathrm{N}, C_{2}=22 / \mu \mathrm{m}$ and $t=0.02 \mu \mathrm{m}$. To characterise the time-dependent mechanics of the network, we introduce a time scale $t_{s}$, which is defined according to the type of computations described hereafter. 
Initially, we perform computations where the network is subjected to a step shear strain $\varepsilon_{12}$ at time zero, and the computed stress is compared to that obtained experimentally. Here, $t_{s}$ is the time after the step strain that the stress is measured.

Further, to investigate the influence of model parameters, we perform a parametric study. In this case, $t_{s}$ is the amount of time over which the strain level applied to the network is ramped to $50 \%$. We vary the bond strength and stiffness over two time-scales, as well as the coefficient $\beta$, see Table 1 .

Table 1 Sets of parameters for study

\begin{tabular}{lcccc}
\hline Set & $E_{b}\left[k_{B} T\right]$ & $C_{1}[\mu N]$ & $C_{2}[1 / \mu m]$ & $\beta$ \\
\hline Baseline & 2 & $1.2 \cdot 10^{-5}$ & 22 & 1 \\
Red. $E_{b}$ & 0.2 & $1.2 \cdot 10^{-5}$ & 22 & 1 \\
Red. $C_{1}$ & 2 & $1.2 \cdot 10^{-6}$ & 22 & 1 \\
Red. $\beta$ & 2 & $1.2 \cdot 10^{-5}$ & 22 & 0.1 \\
\hline
\end{tabular}

\section{Results}

\subsection{Experimental comparisons}

Experiments on actin networks cross-linked by $\alpha$-actinin have been performed by $\mathrm{Xu}$ et al. (2000). While $\alpha$-actinin tends to favour the formation of filament bundles, the concentration of cross-linker in this study was kept low enough to avoid this, and we therefore deem it appropriate to use for comparison. We compare the computed shear stress to these results over two time scales for varying strain levels, see Figure 5. However, to fit results from the shorter time-scale, $\beta$ must be reduced, in this case set to 0.1 . This might be due to a larger amount of energy available to break bonds - at longer time scales mechanisms such as relaxation due to filament motions might be able to reduce this. As will be seen later, the cross-link dynamics in the region of large strains and short time-scales depends on this factor $\beta$. Observing the results in general, we can deduce that the mechanism for stress-relaxation of cross-linked actin is more complex than merely cross-link debonding, as the computed stress is higher than that obtained experimentally. However, in spite of this, the mechanics of cross-link debonding suggests an explanation for experimentally observed behaviour of cross-linked actin networks, such as strain rate dependence and strain softening.

\subsection{Influence of parameters}

The computed shear stress for the parametric study is shown in Figure 6. Some interesting observations can be made from these computations; first, the response at short enough timescales is independent of bond strength $E_{b}$ - there simply is not enough time to allow for debonding. However, one may speculate that if the bond is extremely weak or in the presence of high temperature, debonding events would still occur due to the presence of these terms in the exponential function of Arrhenius' law. There is still a separation from the more compliant cross-links, however, which is to be expected since the cross-link compliance has been found to influence the macroscopic stiffness of crosslinked actin networks (Kasza et al., 2010; Fallqvist et al., 2014). 
Figure 5 Shear stress computation for two time scales at different shear strain levels (see online version for colours)

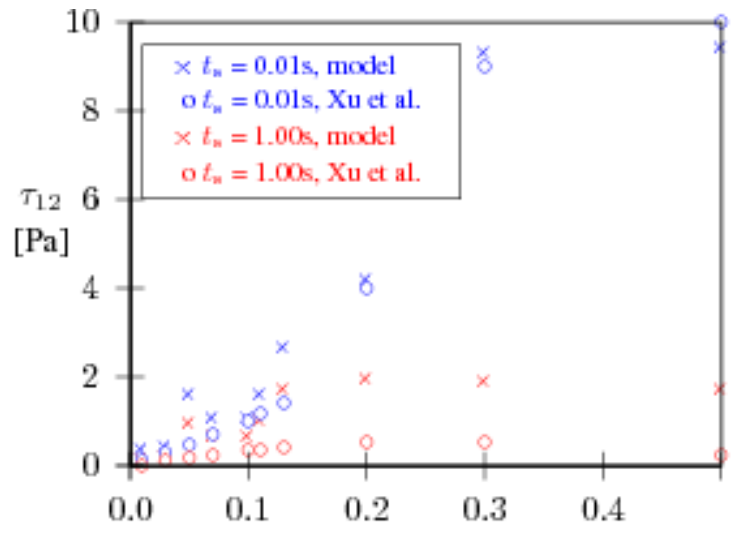

Note: The crosses denote computed results, and the dots are experimental results from $\mathrm{Xu}$ et al. (2000).

Figure 6 Computed shear stress for variation of cross-link properties (see online version for colours)

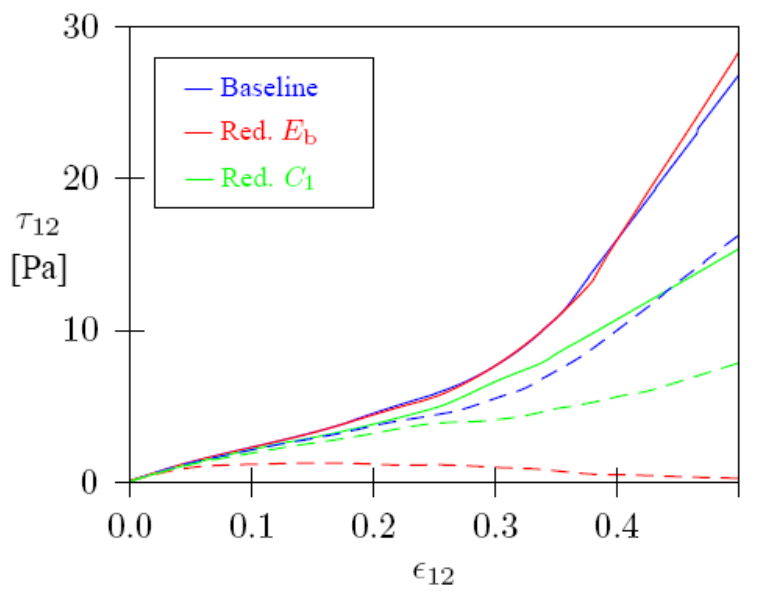

Note: Solid lines are for a time scale of $0.01 \mathrm{~s}$, dashed lines are for $1 \mathrm{~s}$.

Second, a reduction in bond strength yields a softening behaviour at long time scales for large strains (see dashed red line in Figure 6, a behaviour also observed experimentally (Xu et al., 2000).

The coefficient of free bonding energy, $\beta$, is instrumental to the model, and therefore we investigate the influence of $\beta=1$ and $\beta=0.1$ on the large strain-response of our previously defined baseline model, see Figure 7 . It appears that only at large strains and for short time scales, $\beta$ influences the results significantly. When seen in the light of previous results, one may conclude that in general, the bond strength is more important in determining the overall response, and this coefficient can be assumed to be unity. This is also evident when assessing the number of debonded cross-links $n_{d}$ normalised by the total number $n_{\text {tot }}$, see Figure 8 . For short time scales, only at larger strains is there a 
significant difference between computations with $\beta=0.1$ and $\beta=1$, which is a reason for the difference in stress in Figure 7. The dependence on strain-rate found is qualitatively similar to that of Abhilash et al. (2012), in which the propensity for initiation and growth of damage in terms of debonded cross-links is greater for lower strain-rates. It can be noted that the stress-strain relation increases nonlinearly despite an increasing number of debonded cross-links. This is due to the re-orientation of filaments and associated strain hardening of the network itself, found previously in computational studies (Huisman et al., 2007; Fallqvist et al., 2014). The small number of debonded cross-links is apparently not enough to offset this.

Figure 7 Influence of model parameter $\beta$ on the mechanical response, in terms of computed shear stress (see online version for colours)

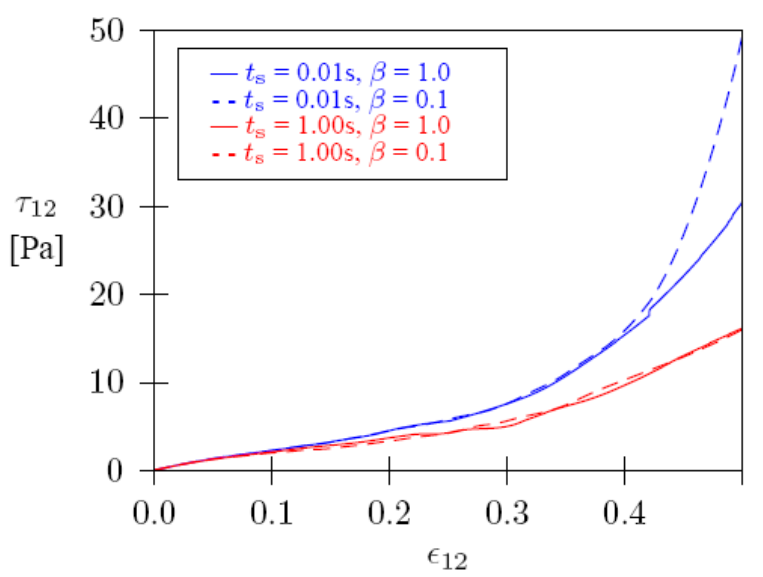

Figure 8 Influence of model parameter $\beta$, in terms of total number of debonded cross-links (see online version for colours)

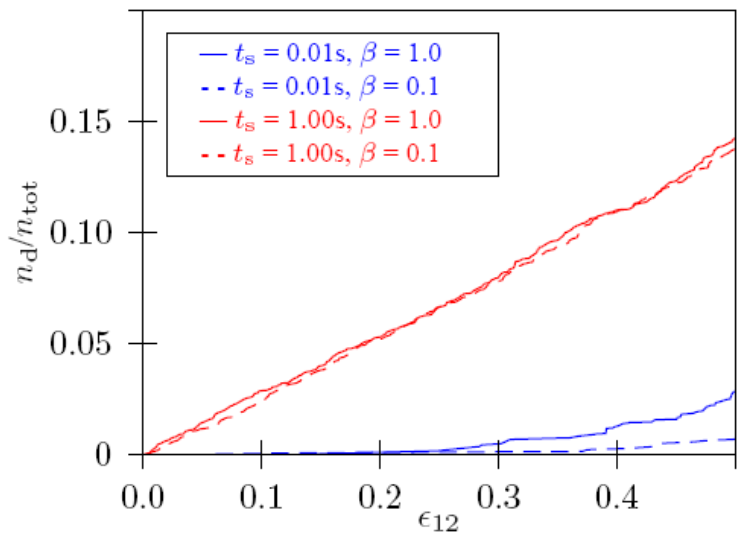




\section{Discussion}

Cross-linked actin networks are significantly different from their entangled counterparts, not only in respect to structure, but also in the way filament interactions affect the macroscopic behaviour. While the viscoelastic behaviour of entangled actin networks can be attributed to the thermal undulations and sliding of filaments, for cross-linked actin networks the additional constraints imposed by the cross-links must also be considered. Disregarding the heterogenous networks of cross-linked bundles formed at certain concentrations of cross-linking proteins, purely cross-linked actin networks have shown themselves to exhibit characteristics such as a strong strain-rate dependence and initial rapid stress relaxation in experiments (Xu et al., 2000; Schmoller et al., 2009; Tseng et al., 2002).

We attribute this partly to the stochastic nature of the cross-links themselves, and propose a model in which a bound cross-link is trapped in an energy well. With an increasing mechanical load, there is a greater probability of a debonding event due to the available strain energy. This is similar to models previously proposed in which Bell's equation is utilised (Abhilash et al., 2012, 2014; Kim et al., 2011), but we hope that this method is a way to develop a more general form of cross-link and filament interaction in which the energy affecting the cross-link dynamics is not solely that of the cross-link tensile force. The drawback of this however, is the difficulty of properly describing the model parameters in terms of physical quantities and these must be seen in a phenomenological sense, for example the parameter $\beta$ described as a fraction of free bonding energy available to break bonds. We can speculate that from this definition, it might depend on factors such as network pre-stress and temperature, especially evident in the inability to find a single value for it to properly predict experimental results.

Of course, the computational framework presented here by no means provides the whole picture - ideally filament-filament interaction (contact and dissipation due to friction) and thermal undulations would be needed, as well as rebinding of the cross-links. However, that is outside the scope of this paper, which focuses on the initial response more than that of long-term stress relaxation, which rebinding kinetics have been found to influence (Kim et al, 2011).

A more accurate fit for longer time-scales can be found by reducing the bond strength, although the initial relaxation then is too fast. Given the uncertainty in measuring the bond strength of a cross-linking protein (for example, $\alpha$-actinin is $2 \pm 1 k_{B} T$ (Biron and Moses, 2004), using a statistical dispersion of these might be more representative of reality.

\section{References}

Abhilash, A., Purohit, P. and Joshi, S. (2012) 'Stochastic rate-dependent elasticity and failure of soft fibrous networks', Soft. Matter., Vol. 8, pp.7004-7016.

Abhilash, A., Zhang, L., Stiefel, J., Purohit, P. and Joshi, S. (2014) 'Predictive maps for stochastic nonaffine stiffening and damage in fibrous networks', Phys. Rev. E, Vol. 3, No. 89, pp.0226,071-0226,079.

Bell, G. (1978) 'Models for specific adhesion of cells to cells', Science, May, Vol. 200, pp.618-627. 
Biron, D. and Moses, E. (2004) 'The effect of $\alpha$-actinin on the length distribution of f-actin', Biophys. J., Vol. 86, No. 5, pp.3284-3290.

Fallqvist, B. and Kroon, M. (2012) 'A chemo-mechanical constitutive model for transiently cross-linked actin networks and a theoretical assessment of their viscoelastic behaviour', Biomech. Model Mechanobio., Vol. 12, No. 2, pp.373-382.

Fallqvist, B., Kulachenko, A. and Kroon, M. (2014) 'Modelling of cross-linked actin networks influence of geometrical parameters and cross-link compliance', J. Theor. Biol., Vol. 350, pp.57-69.

Furuike, S., Ito, T. and Yamazaki, M. (2001) 'Mechanical unfolding of single filamin a (abp-280) molecules detected by atomic force microscopy', FEBS Letters, Vol. 498, pp.72-75.

Gardel, M. and Nakamura, F. (2006) 'Prestressed f-actin networks cross-linked by hinged filamins replicate mechanical properties of cells', PNAS, Vol. 103, No. 6, pp.1762-1767.

Gardel, M., Kasza, K.A., Brangwynne, C., Liu, J. and Weitz, D. (2008) 'Mechanical response of cytoskeletal networks', Methods in Cell Biology, Vol. 89, pp.487-519.

Gardel, M., Shin, J., Mackintosh, F., Mahadevan, L., Matsudaira, P. and Weitz, D. (2004) 'Elastic behavior of cross-linked and bundled actin networks', Science, Vol. 304, No. 5675, pp.1301-1305.

Huisman, E., van Dillen, T., Onck, P. and der Giessen, E.V. (2007) 'Three-dimensional cross-linked f-actin networks: relation between network architecture and mechanical behavior', Phys. Rev. Letters, Vol. 99, pp.208103-1-208103-4.

Kamm, R.D. and Mofrad, M.R.K. (2006) edited by M.R.K. Mofrad, Cytoskeletal Mechanics: Models and Measurements, Cambridge University Press, New York, NY, USA.

Kasza, K., Broedersz, C., Koenderink, G., Lin, Y., Messner, W., Millman, E., Nakamura, F., Stossel, T., Mackintosh, F. and Weitz, D. (2010) 'Actin filament length tunes elasticity of flexibly cross-linked actin networks’, Biophys. J., Vol. 99, No. 4, pp.1091-1100.

Kim, T., Hwang, W. and Kamm, R. (2011) 'Dynamic role of cross-linking proteins in actin rheology’, Biophys. J., Vol. 101, No. 7, pp.1597-1603.

Nunnally, M., Powell, L. and Craig, S. (1980) 'Reconstitution and regulation of actin gel-sol transformation with purified filamin and villin', J. Bio. Chem., Vol. 256, No. 5, pp.2083-2086.

Onck, P., Koeman, T., van Dillen, T. and van der Giessen, E. (2005) 'The origin of stiffening in cross-linked semiflexible networks', Phys. Rev. Letters, Vol. 95, pp.178102-1-178102-4.

Rief, M., Pascual, J., Saraste, M. and Gaub, H. (1999) 'Single molecule force spectroscopy of spectrin repeats: low unfolding forces in helix bundles', J. Mol. Biol., Vol. 286, No. 2, pp.553-561.

Rönnlund, D., Gad, A., Blom, H., Aspenström, P. and Widengren, J. (2013) 'Spatial organization in metastasizing cells', Cytometry Part A, Vol. 83, No. 9, pp.855-865.

Schmoller, K., Lieleg, O. and Bausch, A. (2009) 'Structural and viscoelastic properties of actin/filamin networks: cross-linked versus bundled networks', Biophys. J., Vol. 97, No. 1, pp.83-89.

Tseng, Y., Schafer, B., Almo, S. and Wirtz, D. (2002) 'Functional synergy of actin filament cross-linking proteins', J. Bio. Chem., Vol. 277, No. 28, pp.25,609-25,616.

Wachsstock, D.H., Schwarz, W.H. and Pollard, T.D. (1993) 'Affinity of $\alpha$-actinin for actin determines the structure and mechanical properties of actin filament gels', Biophys. J., Vol. 65, No. 1, pp.205-214.

Wachsstock, D.H., Schwarz, W.H. and Pollard, T.D. (1994) 'Cross-linker dynamics determine the mechanical properties of actin gels', Biophys. J., March, Vol. 66, pp.801-809.

Xu, J., Tseng, Y. and Wirtz, D. (2000) 'Strain hardening of actin filament networks', J. Bio. Chem., Vol. 275, No. 46, pp.35,886-35,892. 